Proceedings of the 1996 IEEE

International Conference on Robotics and Automation

Minneapolis, Minnesota - April 1996

\title{
On Calculation of Optimal Paths with Constrained Curvature: The Case of Long Paths*
}

\author{
Andrei M. Shkel and Vladimir J. Lumelsky \\ University of Wisconsin-Madison \\ Madison, Wisconsin 53706, USA
}

\begin{abstract}
Given two points in the plane, each with the prescribed direction of motion, the question being asked is to find the shortest smooth path of bounded curvature that joins them. The classical result by Dubins [1] that is commonly used gives a sufficient set of paths which is guaranteed to contain the shortest path; the latter is then found by explicitly calculating every path in the set. In this paper we show that in the case when the distance between the two points is above some minimum, the solution sought can be found via a simple classification scheme. Besides computational savings (essential, for example, in real-time motion planning), this result sheds light on the nature of factors affecting the length of paths in the Dubins's problem.
\end{abstract}

\section{INTRODUCTION.}

Consider two points in the plane, $P\left(t_{0}\right)$ and $P\left(t_{f}\right)$ - called the initial and final point - each associated with its own orientation angle, $\alpha$ and $\beta$, respectively. Each orientation angle defines the prescribed direction of motion at the corresponding point; the combinations $\left(P\left(t_{0}\right), \alpha\right)$ and $\left(P\left(t_{f}\right), \beta\right)$ are called the initial and final configuration, respectively (see Figure 1). Given the configurations $\left(P\left(t_{0}\right), \alpha\right)$ and $\left(P\left(t_{f}\right), \beta\right)$ (which together constitute the boundary conditions), the problem is to find the shortest path between $P\left(t_{0}\right)$ and $P\left(t_{f}\right)$, such that it would start and end in the directions $\alpha$ and $\beta$, respectively, and satisfy a constraint on the path curvature, $\rho \leq 1 / R$, where $R$ is the minimal radius of turning.

This kind of tasks appear in numerous applications, such as when joining pieces of railways [2] or planning two- and three-dimensional pipe networks. In robotics this problem plays a central role in most of the nonholonomic motion planners proposed so far $[3,4,5]$. We consider the case - called here the Long Paths Case - when the distance $d$ between points $P\left(t_{0}\right)$ and $P\left(t_{f}\right)$ is sufficiently large (see the definition below; the complementing case,

*This work was supported by the Sea Grant Program (National Oceanic and Atmospheric Administration, US Dept of Commerce, grant NA46RG048), and by DOE (Sandia Labs) Grant 18-4379C. for small $d$, requires a somewhat different handling and is not included here).

The complete solution to this problem was first reported in an elegant paper [1] by Lester Dubins in 1957. He showed that any geodesic (i.e. the shortest path) consists of exactly three path segments and presents a sequence $C C C$ or $C S C$, where $C$ (for "circle") is an arc of radius $R$, and $S$ (for "straight") is a line segment. For a given orientation angle, each arc $C$ has two options - turning left or right. Denote those $r$ and $l$, respectively, and the line segment by $s$. Then the Dubins set, $\mathcal{D}$, includes six paths, or words, $\mathcal{D}=\{l s l, r s r, r s l, l s r, r l r, l r l\}$. Furthermore, Dubins's theorem states that in order to be a candidate for the optimal path, each arc has to be of the minimal allowed radius $R$.

Using advanced calculus, this result of Dubins was later proved by J. Reeds and L. Shepp [6]; they were also able to obtain further results for a more complex case with motion reversals. Finally, J. Boissonnat et al. [7] proved this result from the standpoint of optimal control, by making use of the powerful Pontryagin's optimality principle [8].

The actual implementation of the Dubins's result for the shortest path calculation requires an explicit calculation of the lengths of all arcs and straight line segments in the Dubins set, and then choosing the shortest of the six members of the set. A natural question here is whether the exhaustive calculation of the set can be avoided or reduced via some implicit classification of the words in the set.

Another motivation behind this work is to produce an efficient kernel calculation scheme which could be used on a continuous basis in real-time sensor-based systems with dynamics, where the time constraints on calculations are quite severe.

The following analysis of the Dubins set is based on the notion of equivalency groups formed by the pairs of orientation angles depending on their angular quadrant. Each such group consists of a few classes of paths, such that any path is equivalent, up to an orthogonal transformation, to any other path in the same group. This means that the optimal path analysis can be reduced to 


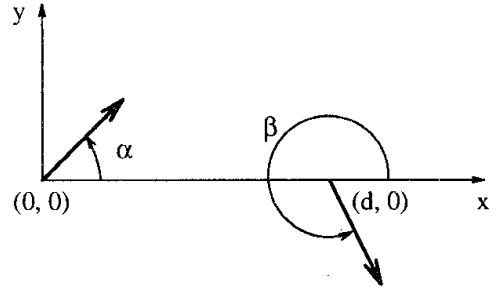

Fig. 1. Shown are the coordinate system, the initial $\left(P\left(t_{0}\right), \alpha\right)$ and the final $\left(P\left(t_{f}\right), \beta\right)$ configurations.

fewer elements. Furthermore, a simple classification of the equivalency groups can either produce the optimal path directly, thus eliminating any explicit calculation of paths, or reduce the number of candidates to consider.

Below, $d$ is the distance between the initial, $P\left(t_{0}\right)$, and final, $P\left(t_{f}\right)$, positions. A rectangular coordinate system $(x, y)$ is chosen such that its origin is $P\left(t_{0}\right)=(0,0)$ and the positive direction of the $x$-axis is toward $P\left(t_{f}\right)$, Figure 1 . The coordinates of point $P\left(t_{f}\right)$ are thus $P\left(t_{f}\right)=(d, 0)$. The initial and final orientation angles, $\alpha$ and $\beta$, are measured counter-clockwise from the positive direction of $x$ axis. Without loss of generality, assume a unit radius of the minimum turning circle, $R=1$ (any other $R$ can be reduced to 1 by the scaling $d=D / R$, where $D$ is the actual distance between $P\left(t_{0}\right)$ and $\left.P\left(t_{f}\right)\right)$.

Denote the initial and final arcs in the Dubins set as $C_{i l}, C_{i r}, C_{f l}, C_{f r}$ ( $r$ and $l$ stand for "right" and "left"). Then, in more precise terms, the case being studied is when the distance $d$ satisfies $\left\{C_{i l} \cup C_{i r}\right\} \cap\left\{C_{f l} \cup C_{f r}\right\}=\emptyset$. This covers all cases when $d>4 R$ and many cases of smaller $d$ (see Figure 4 and Proposition 3 ).

The set of admissible paths is studied in Section II. The summary of the approach used is given in Section III, followed by the statement of the main result in Section IV and its further analysis in Section V. Some proofs omitted due to space limitations can be found in [9].

\section{ADMISSIBLE PATHS AND THEIR SPECIFICATIONS}

Following [1], an admissible path is defined as a continuously differentiable curve which is either (i) an arc of the circle of radius 1 , followed by a line segment, followed by another arc of the circle of radius 1 ; or (ii) a sequence of three arcs of circles of radius 1; or (iii) a subpath of path of type (i) or (ii).

Assume we have an admissible path $\gamma(t)$ from the Dubins set, between the initial, $\left(P\left(t_{0}\right), \phi\left(t_{0}\right)\right)$, and final, $\left(P\left(t_{f}\right), \phi\left(t_{f}\right)\right)$, configurations, where $\phi\left(t_{0}\right)=\alpha$ and $\phi\left(t_{f}\right)=\beta$. The length of the admissible path $\gamma$ : $\left(P\left(t_{0}\right), \phi\left(t_{0}\right)\right) \rightarrow\left(P\left(t_{f}\right), \phi\left(t_{f}\right)\right)$ is then defined as $\mathcal{L}(\gamma)=$ $t_{f}-t_{0}$.

To specify the admissible paths, we will use the following elementary transformations. Given the initial and final configurations, $\left(x\left(t_{0}\right), y\left(t_{0}\right), \alpha\right) \in R^{3}$ and $\left(x\left(t_{f}\right), y\left(t_{f}\right), \beta\right) \in R^{3}$, we introduce three elementary motions: rotation to the left, rotation to the right (both along a circle of radius 1 ), and straight line motion. To specify a transformation, there are three corresponding operators, $L_{t}$ (for left), $R_{t}$ (for right), $S_{t}$ (for straight):

$$
\begin{aligned}
L_{t} & =R^{3} \rightarrow R^{3}, \\
R_{t} & =R^{3} \rightarrow R^{3}, \\
S_{t} & =R^{3} \rightarrow R^{3}
\end{aligned}
$$

where index $t$ indicates that the corresponding motion is along the segment of length $t$.

By applying an elementary motion operator, an arbitrary point $(x, y, \phi) \in R^{3}$ is transformed into its corresponding image point in $R^{3}$. The formulas for the elementary transformations are given by

$$
\begin{aligned}
L_{t}(x, y, \phi)= & (x+\sin (\phi+t)-\sin \phi, \\
& y-\cos (\phi+t)+\cos \phi, \phi+t) \\
R_{t}(x, y, \phi)= & (x-\sin (\phi-t)+\sin \phi, \\
& y+\cos (\phi-t)-\cos \phi, \phi-t) \\
S_{t}(x, y, \phi)= & (x+t \cos \phi, y+t \sin \phi, \phi)
\end{aligned}
$$

With these elementary transformations, any word (path) from the Dubins set $\mathcal{D}$ can be expressed in terms of the corresponding equations. In the coordinate system as chosen, the initial configuration of each path is at $(0,0, \alpha)$ and the final configuration at $(d, 0, \beta)$. For example, the path $l_{t} r_{p} l_{q}$ that starts at the configuration $(0,0, \alpha)$ must end at $L_{q}\left(R_{p}\left(L_{t}(0,0, \alpha)\right)\right)=(d, 0, \beta)$. The length $\mathcal{L}$ of each path can be defined as the sum of the segments $t, p$ and $q$,

$$
\mathcal{L}=t+p+q
$$

We would like to build a scheme for classifying the Dubins set $\mathcal{D}=\{l s l, r s r, r s l, l s r, r l r, l r l\}$, with the purpose of obtaining the optimal solution from the classification, rather than from an explicit calculation and comparison of all six words. To this end, we now derive the operator equations defining each word in the set $\mathcal{D}$.

$$
\begin{aligned}
\text { lsl : } & L_{q}\left(S_{p}\left(L_{t}(0,0, \alpha)\right)\right)=(d, 0, \beta) ; \\
\text { rsr : } & R_{q}\left(S_{p}\left(R_{t}(0,0, \alpha)\right)\right)=(d, 0, \beta) ; \\
\text { lsr : } & R_{q}\left(S_{p}\left(L_{t}(0,0, \alpha)\right)\right)=(d, 0, \beta) ; \\
r s l: & L_{q}\left(S_{p}\left(R_{t}(0,0, \alpha)\right)\right)=(d, 0, \beta) ; \\
\text { rl : } & R_{q}\left(L_{p}\left(R_{t}(0,0, \alpha)\right)\right)=(d, 0, \beta) ; \\
\text { lrl : } & L_{q}\left(R_{p}\left(L_{t}(0,0, \alpha)\right)\right)=(d, 0, \beta) ;
\end{aligned}
$$

By solving these operator equations, we can find segments $t, p$, and $q$ for each word from the Dubins set. According to (2), the sum of $t, p$, and $q$ defines the length of 
the corresponding path. For example, the length of the path $r s l$, as a function of $\alpha, \beta$ and $d$, is given by

$$
\begin{gathered}
\mathcal{L}_{r s l}=p+q+t=2 t+p-\alpha+\beta(\bmod 2 \pi)= \\
\alpha+\beta(\bmod 2 \pi)-2 \arctan \frac{\cos \alpha+\cos \beta}{d-\sin \alpha-\sin \beta}+ \\
2 \arctan \frac{2}{p}+p
\end{gathered}
$$

where

$$
p=\sqrt{d^{2}-2+2 \cos (\alpha-\beta)-2 d \sin \alpha-2 d \sin \beta}
$$

\section{EQUIVALENCE GROUPS}

We are now ready to turn to the classification of the Dubins set $\mathcal{D}$. With the orientations $\alpha$ and $\beta$ defined as in Figure 1, we divide the whole range of possible orientations into 4 regions (quadrants). Region 1 corresponds to the range $[0, \pi / 2]$, region $2-$ to $[\pi / 2, \pi]$, region 3 - to $[\pi, 3 \pi / 2]$, and region 4 - to the range $[3 \pi / 2,2 \pi]$. Since each of $\alpha$ or $\beta$ can be in any of the four regions, together we have 16 different combinations of possible orientation regions.

These 16 cases can be shown to reduce to only 6 independent combinations. Each of the remaining 10 combinations presents an orthogonal transformation of one of the 6 and thus has the same topology and the same path length. This fact helps reduce the number of path candidates that have to be considered to find the optimal solution. (Recall that the set of candidates to consider is further reduced due to the constraint of the Long Paths Case, $\left.\left\{C_{i l} \cup C_{i r}\right\} \cap\left\{C_{f l} \cup C_{f r}\right\}=\emptyset\right)$.

We represent these 16 combinations of $(\alpha, \beta)$ by a $4 \times 4$ matrix, $\left\{a_{i j}\right\}$, where index $i$ corresponds to the initial, and index $j$ - to the final orientation. For example, the case $\alpha \in[0, \pi / 2]$ and $\beta \in[\pi / 2, \pi]$ corresponds to the element $a_{12}$ of the matrix $\left\{a_{i j}\right\}$. That is, element $a_{i j}$ describes the class of all possible paths whose initial and final orientation angles belong to the corresponding quadrants.

Dubins's theorem says that each candidate for the optimal path in set $\mathcal{D}$ must start with a piece of circle and end with a piece of circle (of radius $R=1$, see above). Depending on the word in $\mathcal{D}$, the initial (and the final) circle can turn either left or right; we denote those $C_{i l}$ and $C_{i r}\left(C_{f l}\right.$ and $\left.C_{f r}\right)$, respectively.

Later we will make use of the following proposition about equivalent orientations:

Proposition 1 for any path connecting two points, $P\left(t_{0}, \alpha\right), P\left(t_{f}, \beta\right)$, where $(\alpha, \beta)$ are the initial and final orientation angles, there exist three other paths between $P\left(t_{0}\right)$ and $P\left(t_{f}\right)$ which are equivalent to the original path up to an orthogonal transformation. Their corresponding orientation angles are $(-\alpha,-\beta),(\beta, \alpha)$ and $(-\beta,-\alpha)$.

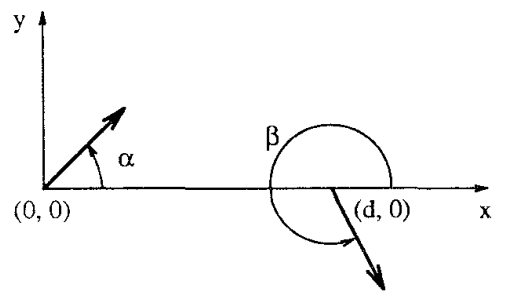

Fig. 2. Path $I A_{1} A_{2} F$ is equivalent to the paths $I B_{1} B_{2} F, I D_{1} D_{2} F, I E_{1} E_{2} F$.

As an example, take one path, say $I A_{1} A_{2} F$, in Figure 2. Then, paths $I B_{1} B_{2} F, I D_{1} D_{2} F$, and $I E_{1} E_{2} F$ can be obtained from $I A_{1} A_{2} F$ by applying the following orthogonal transformations:

$$
\begin{array}{r}
I A_{1} A_{2} F \stackrel{R_{G H}}{\longrightarrow} I B_{1} B_{2} F \\
I A_{1} A_{2} F \stackrel{R_{I F}}{\longrightarrow} I E_{1} E_{2} F \\
I A_{1} A_{2} F \stackrel{R_{G H}}{\longrightarrow} I B_{1} B_{2} F \stackrel{R_{I F}}{\longrightarrow} I D_{1} D_{2} F
\end{array}
$$

where $R_{G H}$ and $R_{I F}$ are the operators of reflection with respect to the lines $G H$ and $I F$, respectively. Clearly, if the orientation angles of path $I A_{1} A_{2} F$ are $\alpha, \beta$, $I A_{1} A_{2} F[\alpha, \beta]$, then

$$
\begin{array}{r}
I A_{1} A_{2} F[\alpha, \beta] \simeq I B_{1} B_{2} F[-\beta,-\alpha] \simeq \\
I D_{1} D_{2} F[\beta, \alpha] \simeq I E_{1} E_{2} F[-\alpha,-\beta]
\end{array}
$$

(here " $\simeq "$ is the sign of equivalence). It follows from this proposition that the matrix $\left\{a_{i j}\right\}$ can be divided into few equivalence groups. The following statement holds:

Proposition 2 Matrix $\left\{a_{i j}\right\}$ can be divided into six independent equivalence groups: (1) $a_{11} \simeq a_{44}$, (2) $a_{12} \simeq$ $a_{21} \simeq a_{34} \simeq a_{43}$, (3) $a_{13} \simeq a_{24} \simeq a_{31} \simeq a_{42}$, (4) $a_{14} \simeq a_{41}$, (5) $a_{22} \simeq a_{33}$, and (6) $a_{23} \simeq a_{32}$.

For example, for the equivalence group 2, for any path of class $a_{12}$ there exists an equivalent path in each of the classes $a_{21}, a_{34}$, and $a_{43}$.

By choosing one representative from each equivalence group in matrix $\left\{a_{i j}\right\}$, we define a basis set $\mathcal{B}$ of matrix $\left\{a_{i j}\right\}$ - a set of six mutually independent classes of orientation pairs. Therefore, the number of orientation pairs to be analyzed for the optimal solution can be reduced from 16 to 6 . Notice that the basis set is not unique since its members can be chosen in various ways.

\section{THE MAIN RESULT}

The main result of this work is in showing that in the case at hand the problem of finding the shortest smooth path between two configurations can be solved largely without an explicit calculation of the paths involved, using a simple 
logical scheme. In the scheme, all possible paths are first classified into groups, such that the membership in a group eventually defines the optimal solution.

The resulting classification is summarized in the table in Figure 3. The procedure for finding the optimal solution is thus simple: find the quadrants of the given orientation angles $(\alpha, \beta)$, and then find the corresponding element in the table Figure 3 . Note that in some cases a single solution appears directly, whereas in other cases two candidates have to be considered. As an example, the set $\alpha \in[0, \pi / 2]$ and $\beta \in[0, \pi / 2]$ (element $a_{11}$ of matrix $\left\{a_{i j}\right\}$ ) represents the former type: the optimal path solution is the word $r s l$. The element $a_{12}$ is an example of the latter type: knowing the quadrant is not sufficient to pinpoint the optimal solution, and further processing of the subset $(r s l, r s r, l s r)$ is necessary (refer to [9] for details).

\begin{tabular}{|c|c|c|c|c|}
\hline $\begin{array}{l}\text { Final Quadrant } \\
\text { Initial Quadrant }\end{array}$ & 1 & 2 & 3 & 4 \\
\hline 1 & rsl & $\begin{array}{c}\text { rsl or } \\
\{r s r \text { or } 1 s r\}\end{array}$ & $\{$ rsr or $1 \mathrm{sr}\}$ & $\{\mathrm{rsr}$ or $1 \mathrm{sr}\}$ \\
\hline 2 & $\begin{array}{c}\text { rsl or } \\
\{l s l \text { or } l s r\}\end{array}$ & $\begin{array}{c}\text { rsl or } \\
\{\mathrm{rsr} \text { or } 1 \mathrm{sl}\}\end{array}$ & rsr & $\{$ rsr or rsl $\}$ \\
\hline 3 & $\{1 \mathrm{sl}$ or $1 \mathrm{sr}\}$ & 1sl & $\begin{array}{c}1 \mathrm{sr} \text { or } \\
\{\mathrm{lsl} \text { or } \mathrm{rsr}\}\end{array}$ & $\begin{array}{c}\text { lsr or } \\
\{\mathrm{rsr} \text { or } \mathrm{rsl}\}\end{array}$ \\
\hline 4 & $\{1 \mathrm{sr}$ or $\mathrm{rsl}\}$ & $\{\mathrm{lsl}$ or $\mathrm{rsl}\}$ & $\begin{array}{c}\text { lsr or } \\
\{1 \mathrm{sl} \text { or } \mathrm{rsl}\}\end{array}$ & lsr \\
\hline
\end{tabular}

Fig. 3. The table of solutions.

\section{The Method for Path Classification}

Building the scheme for classifying the Dubins set involves the as following steps:

(a) Find the condition of no intersection of the union $\left\{C_{i l} \cup C_{i r}\right\}$ with the union $\left\{C_{f l} \cup C_{f r}\right\}$.

(b) Justify what simplifications in the set of candidates for the optimal solutions are implied if condition (a) is satisfied.

(c) Specify the basis set $\mathcal{B}$ and the optimal solutions for all elements of the set.

(d) Using proposition 1 , show how to find the optimal solution for the remaining elements of $\left\{a_{i j}\right\}$.

We demonstrate the scheme on one basis set; take, for example, the basis set $\mathcal{B}=\left\{a_{11}, a_{12}, a_{13}, a_{14}, a_{22}, a_{23}\right\}$.

Proposition $3 \quad\left\{C_{i l} \cup C_{i r}\right\} \cap\left\{C_{f l} \cup C_{f r}\right\}=\emptyset$ if and only if

$d>\sqrt{4-(|\cos \alpha|+|\cos \beta|)^{2}}+|\sin \alpha|+|\sin \beta|$

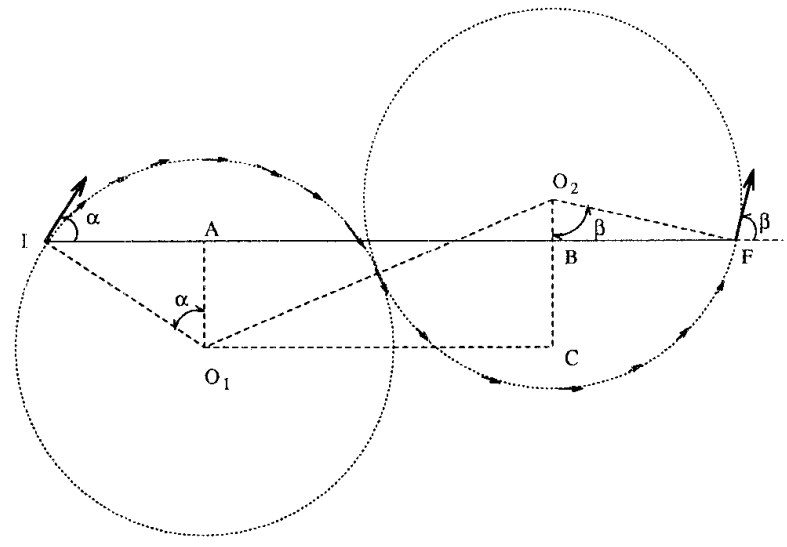

Fig. 4. The $r l$-case; here both $\alpha$ and $\beta$ are in the first quadrant.

To prove this necessary and sufficient condition for the Long Paths Case, consider the case when the set $\left\{C_{i l} \cup C_{i r}\right\}$ is tangent to the set $\left\{C_{f l} \cup C_{f r}\right\}$. Take, for instance, the case $a_{11}$ ( $\alpha$ and $\beta$ are in the first quadrant, Figure 4). Assume a unit radius, $R=1$. Here two circle arcs have a common tangent, and so $|I F|=|I A|+|A B|+|B F|$. From $\triangle I A O_{1}:|I A|=\sin \alpha$ and $\left|O_{1} A\right|=\cos \alpha$. From $\triangle F B O_{2}: \quad|B F|=\sin \beta$ and $\left|O_{2} B\right|=\cos \beta$. From $\triangle O_{1} O_{2} C:\left|O_{1} O_{2}\right|=2,\left|O_{2} C\right|=\left|O_{2} B\right|+\left|O_{1} A\right|$, and therefore $\left|O_{1} C\right|=\sqrt{4-(\cos \alpha+\cos \beta)^{2}}$. Summing up for: $|I A|+|A B|+|B F|$, obtain the expression for distance $|I F|$, which is a condition for a common tangent for the right initial circle and left final circle. In general, the expression $\sqrt{4-(|\cos \alpha|+|\cos \beta|)^{2}}+|\sin \alpha|+|\sin \beta|=d$ covers all possible cases for two circles with a common tangent point.

Proposition 4 If the boundary conditions are such that $\left\{C_{i l} \cup C_{i r}\right\} \cap\left\{C_{f l} \cup C_{f r}\right\}=\emptyset$ then the word $C C C$ cannot be the optimal solution.

To show this, consider the set $\mathcal{B}$ of independent orientations. We have to show that for any possible pair of orientations (that is, for any element from $\mathcal{B}$ ) there exists a path of type $C S C$ which is shorter than the path $C C C$. An example that illustrates this point is shown in Figure 5 .

Start with the element $a_{11} \in \mathcal{B}$, for which $\alpha$ and $\beta$ are in the first quadrant, Figure 5 . If the shortest word were of type $C C C$, it would be either $r l r$ or $l r l$. Consider the case when $C C C$ is of the form $r l r$ (for the case $l r l$ the proof is similar). We will now show that for any word of the form $r l r$ there exists a word of type $C S C$ which is shorter.

Build a common tangent line, $\left(L_{1}, L_{2}\right)$, for the right circle of the initial position and the left circle of the final 


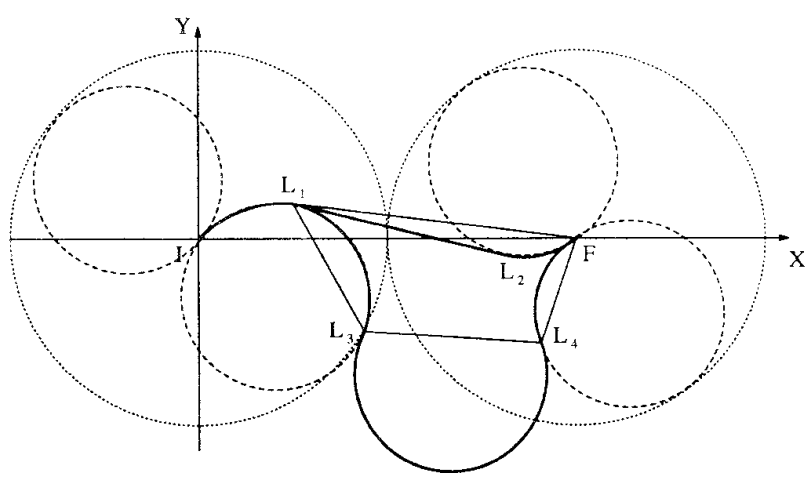

Fig. 5. This example illustrates that if the initial and final circles do not intersect, then the word of type $C C C$ cannot be the optimal solution.

position, Figure 5. Then, draw a circle tangent to the initial and final circles, to complete the $C C C$ path $r l r$. If the tangent points $L_{3}$ and $L_{4}$ are above the curve $L_{1} L_{2} F$ then the middle arc of the word $r l r$ will be less than $\pi$ and can be shortened [1]. If $L_{3}$ and $L_{4}$ are below the curve $L_{1} L_{2} F$ (as in Figure 5), connect points $L_{1}, L_{3}, L_{4}, F$ by straight line segments. Since the straight line is the shortest distance between two points in the plane, we have $L_{1}^{-} L_{3}<L_{1} L_{3}, L_{3} L_{4}<L_{3} L_{4}, L_{4}^{-} F<L_{4} F$.

Summing up these inequalities, obtain $L_{1}^{-} L_{3}+{ }_{L_{3}}^{-} L_{4}+$ $\widetilde{L_{4} F}<L_{1} L_{3}+L_{3} L_{4}+L_{4} F$. Since curve $L_{1} L_{2} F$ is shorter than $L_{1} L_{3}+L_{3} L_{4}+L_{4}^{-F}$, then curve $I L_{1} L_{2} F$ is shorter than the curve $I L_{1} L_{3} L_{4} F$. Notice that this fact is independent of the boundary conditions, as long as the initial and final circles are not intersecting and the curves in question correspond to the element $a_{11}$. The following proposition holds:

Proposition 5 For the Long Paths Case, $\left\{C_{i l} \cup C_{i r}\right\} \cap$ $\left\{C_{f l} \cup C_{f r}\right\}=\emptyset$, the optimal solution corresponding to the element $a_{11}$ is $r s l$.

Note that the number of the candidate curves for the optimal solution in set $\mathcal{D}$ is now reduced to lsl, lsr, rsl, rsr - the curves of type $C C C$ are excluded from the consideration due to Proposition 4. For the three words, $l s l, l s r, r s r$, the $x$ coordinate goes outside the range $0 \leq x \leq d$. Take the length of the curve $l s$ as the lower bound on the length of the words $l s l$ and $l s r$ ( $l s$ is a subword of words $l s l$ or $l s r$ with $\alpha=\pi / 2$ ). Notice, the word $r s r$ cannot be considered as a candidate for the optimal solution of the element $a_{11}$.

We claim that the upper bound on the length of words for which the $x$ coordinate is in the range $0 \leq x \leq d$ is the word $r s l$ with $\alpha=\beta=\pi / 2$. Indeed, from Section II, $\partial \mathcal{L}_{r s l} / \partial \alpha>0$ and $\partial \mathcal{L}_{r s l} / \partial \beta>0$. Therefore, the maximum of $\mathcal{L}_{r s l}$ in this region will occur when $\alpha$ and $\beta$ are equal to $\pi / 2$.

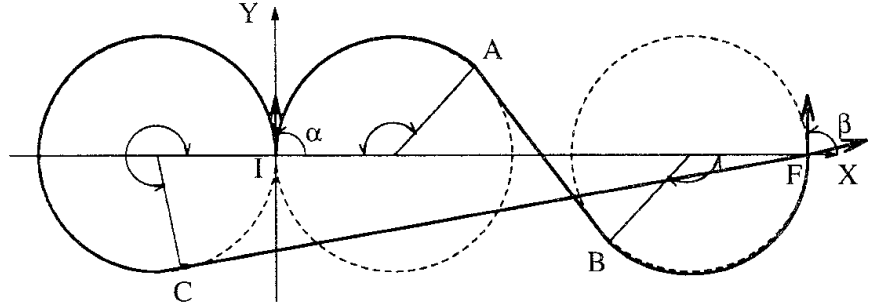

Fig. 6. Optimal solution; here both $\alpha$ and $\beta$ are in the first quadrant.

To prove that the optimal solution for the words within the range $0 \leq x \leq d$ is $r s l$, we have to show that the lower bound on the path length in the Dubins subset $\{l s l, r s r, l s r\}$ is bigger than the upper bound on the word $r s l$. This case is illustrated in Figure 6.

Note that if $\alpha$ and $\beta$ are in the first quadrant then the upper bound for $\mathcal{L}_{r s l}$ will be limited by

$$
\max _{\alpha, \beta \in[0, \pi / 2]} \mathcal{L}_{r s l} \leq \sqrt{d^{2}-4 d}+2 \pi
$$

and the lower bound for the remaining candidates for the optimal solution $\mathcal{L}_{l s l}, \mathcal{L}_{l s r}$, and $\mathcal{L}_{r s r}$ is,

$$
\min _{\alpha, \beta \in[0, \pi / 2]}\left\{\mathcal{L}_{l s l}, \mathcal{L}_{l s r}, \mathcal{L}_{r s r}\right\} \geq \sqrt{d^{2}+2 d}+\frac{3}{2} \pi
$$

In order to prove that the optimal solution corresponding to the element $a_{11}$ is $r s l$, we need to show that

$$
\min _{\alpha, \beta}\left\{\mathcal{L}_{l s l}, \mathcal{L}_{l s r}, \mathcal{L}_{r s r}\right\}-\max _{\alpha, \beta} \mathcal{L}_{r s l}>0
$$

It is easy to see that (4) holds if $\sqrt{d^{2}+2 d}-\sqrt{d^{2}-4 d}-$ $\pi / 2>0$ : move $\pi / 2$ to the right side of the inequality and multiply both sides by the positive expression $\sqrt{d^{2}+2 d}+$ $\sqrt{d^{2}-4 d}$. The result is $6 d>\pi \sqrt{d^{2}+2 d}$, which is true if $d^{2}-4 d>0-$ precisely the case we are interested in. This completes the proof of inequality (4) and of the claim that the optimal solution for element $a_{11}$ is $r s l$.

The proofs for the remaining elements of the set $\mathcal{B}$ are similar and can be found in [9]. The results of this analysis for the remaining elements of set $\mathcal{B}$ are summarized in the following proposition:

Proposition 6 The elements of the basis set of independent orientations $\mathcal{B}=\left\{a_{11}, a_{12}, a_{13}, a_{14}, a_{22}, a_{23}\right\}$ have the following optimal solutions:

$a_{11} \mapsto\{r s l\} ; \quad a_{12} \mapsto\{r s l$ or $\{r s r$ or $l s r\}\} ;$

$a_{13} \mapsto\{r s r$ or $l s r\} ; a_{14} \mapsto\{r s r$ or $l s r\}$

$a_{22} \mapsto\{r s l$ or $\{r s r$ or $l s l\}\} ; a_{23} \mapsto\{r s r\}$.

For example, $a_{12} \mapsto\{r s l$ or $\{r s r$ or $l s r\}\}$ means that if the initial and final orientations are $\alpha \in[0, \pi / 2]$ and $\beta \in$ $[\pi / 2, \pi]$ (which corresponds to the element $a_{12}$ of matrix 
$\left.\left\{a_{i j}\right\}\right)$, then the corresponding optimal solution is one of the words $r s l, r s r, l s r$. Further classification can then be applied: continuing with the class $a_{12}$, depending on the sign of the function $Q(\alpha, \beta, d)=-1+\cos (\alpha-\beta)+d \sin \alpha$, some words can be eliminated from the set of candidates. Namely, if $Q>0$ then the word $l s r$ can be eliminated; otherwise, word $r s r$ is eliminated. Other classifications and their proofs can be found in [9].

Proposition 6 can be further extended based on the Proposition 1. The corresponding results are summarized in the following proposition, and also in the table in Figure 3 .

Proposition 7 1) Since $a_{11} \simeq a_{44}$ and the optimal solution for $a_{11}$ is $r s l$, then the optimal solution for $a_{44}$ is lsr.

2) Since $a_{12} \simeq a_{21} \simeq a_{34} \simeq a_{43}$ and the optimal solution for $a_{12}$ is $\{r s l$ or $\{r s r$ or $l s r\}\}$, then the other optimal solutions are $a_{21} \mapsto\{r s l$ or $\{l s l$ or $l s r\}\} ; a_{34} \mapsto\{l s r$ or $\{r s r$ or $r s l\}\} ; a_{43} \mapsto\{l s r$ or $\{l s l$ or $r s l\}\}$.

3) Since $a_{13} \simeq a_{24} \simeq a_{31} \simeq a_{42}$ and the optimal solution for $a_{13}$ is $\{r s r$ or lsr $\}$, the other optimal solutions are $a_{24} \mapsto\{r s r$ or $r s l\} ; a_{31} \mapsto\{l s l$ or $l s r\} ; a_{42} \mapsto\{l s l$ or $r s l\}$.

4) Since $a_{14} \simeq a_{41}$ and the optimal solution for $a_{14}$ is $\{r s r$ or $l s r\}$, the optimal solution for $a_{41}$ is $\{l s r$ or $r s l\}$.

5) Since $a_{22} \simeq a_{33}$ and the optimal solution for $a_{22}$ is $\{r s l$ or $\{r s r$ or $l s l\}\}$, then the optimal solution for $a_{33}$ is $\{l s r$ or $\{l s l$ or $r s r\}\}$.

6) Since $a_{23} \simeq a_{32}$ and the optimal solution for $a_{23}$ is rsr, the optimal solution for $a_{32}$ is lsl.

\section{CONCLUSION}

The central idea suggested in this paper for finding the shortest path between two points is that of classification of path candidates, as opposed to their explicit calculation and comparison. The resulting savings in calculations makes it practical to do continuous real-time optimization of the path. For example, for a car-like mobile robot this will translate into a simple scheme executed at each step: find the element in the table Figure 3 that corresponds to the current configuration, and then move according to the corresponding solution word; if the solution is not unique, choose the shortest one from the reduced set of candidates.

Perhaps more significantly, the proposed scheme can be used for path classification in the case when there are no restrictions on the path smoothness. In this case, the sufficient set for the optimal solution is much larger - it contains 48 candidates [6], thus leading to more serious computational savings.

\section{REFERENCES}

[1] L. E. Dubins. On curves of minimal length with a constraint on everage curvature, and with prescribed initial and terminal positions and tangents. American Journal of Mathematics, 79:497-516, 1957.

[2] M. Krein and A. Nudel'man. "The Markov moment problem and extremal problems". The american mathematical Society, 1977.

[3] P. Jacobs and J. Canny. Planning smooth paths for mobile robots. Proc. IEEE Intern. Conf. on Robotics and Automation, May 1989.

[4] P. Jacobs, J.P. Laumond, and M. Taix. Efficient motion planners for nonholonomic mobile robots. IEEE/RSJ Intern. Workshop On Intelligent Robots and Systems, August 1991. Osaka, Japan.

[5] J. Barraquand and J.-C. Latombe. On nonholonomic mobile robots and optimal maneuvering. In 4th International Symposium on Intelligent Control, 1989. Albany, NY.

[6] J. A. Reeds and L. A. Shepp. Optimal paths for a car that goes both forwards and backwards. Pacific Journal of Mathematics, 14.5:367-393, 1990.

[7] J. Boissonnat, A. Cerezo and J. Leblond. Shortest paths of bounded curvature in the plane. Proc. IEEE Intern. Conf. on Robotics and Automation, May 1992.

[8] L. S. Pontryagin. "The mathematical theory of optimal processes". Interscience Publishers, New York, 1962.

[9] A. Shkel and V. Lumelsky. "Classification of the Dubins Set. I. The case of long paths". Technical Report RL-96001, Robotics Laboratory, University of Wisconsin-Madison, January 1996. 